\title{
Casos prácticos como herramienta educativa en las asignaturas de fisiología humana
}

\section{Silvia Tejada a, Maria del Mar Bibiloni ${ }^{\mathrm{b}}$, David Moranta ${ }^{\mathrm{c}}$, Susana Esteban ${ }^{\mathrm{c}}$, Antoni Sureda $^{\text {b }}$}

${ }^{a}$ Laboratory of Neurophysiology, Department of Biology, IUNICS, University of the Balearic Islands, Ctra Valldemossa km 7.5, Balearic Islands, Spain; y CIBEROBN (Physiopathology of Obesity and Nutrition), E-07122 Palma de Mallorca, Balearic Islands; silvia.tejada@uib.es ${ }^{b}$ Research Group on Community Nutrition and Oxidative Stress, University of Balearic Islands, E-07122 Palma de Mallorca, Balearic Islands y CIBEROBN; mar.bibiloni@uib.es y antoni.sureda@uib.es cLaboratory of Neurophysiology, Department of Biology, IUNICS, University of the Balearic Islands, Ctra Valldemossa km 7.5, Balearic Islands, Spain; david.moranta@uib.es y susana.esteban@uib.es

\begin{abstract}
The education plans of the universities represent a coincidence in the subject of Human Physiology (Physiotherapy, Biology, Biochemistry, Nursing and Medicine degrees), in which teaching for small groups are a good strategy to achieve certain skills that could not be achieved during large groups time. The main methodology followed is the realization of monographic works. During the 2018-2019 academic year, the University of the Balearic Islands awarded a teaching innovation project in which the design and coordination of seminars was proposed through an active learning methodology through the resolution of practical cases that would help students to acquire skills in critical thinking, leadership, emotional intelligence, adaptability and management of new technologies. The students had to solve a supposed clinical case, exposing the normal physiological process and the physiological alteration associated with the proposed case. The results obtained ( $n=174$ students) indicated an improvement in the acquisition of knowledge (average of 8.2) and in the satisfaction (average of 8.1) obtained by the students, who preferred this methodology to monographic expositions or the use of simulators by computer. In conclusion, the methodology contributed was received with a high degree of satisfaction for its innovative application in the small groups.
\end{abstract}

Keywords: project, innovation, teaching, collaborative work, coordination, meaningful learning

\footnotetext{
Resumen

Los planes docentes de diversas titulaciones suponen una coincidencia en la asignatura de Fisiología Humana (grados: Fisioterapia, Biología, Bioquímica y Medicina), en los cuales las horas docentes destinadas a grupos pequeños suponen una buena estrategia para alcanzar determinadas competencias que no se podrían alcanzar con grupos grandes. La realización de trabajos monográficos es la principal metodología seguida. Durante el
} 
curso 2018/2019, la Universidad de las Islas Baleares concedió un proyecto de innovación docente donde se planteó el diseño y la coordinación de la realización de seminarios mediante una metodología activa de aprendizaje a través de resolución de casos prácticos con el objetivo de adquirir destrezas en pensamiento crítico, liderazgo, inteligencia emocional, adaptabilidad y manejo de las nuevas tecnología. Los alumnos debían resolver un supuesto caso clínico, exponiendo el proceso fisiológico normal y la alteración fisiológica asociada al caso propuesto. Los resultados obtenidos ( $n=174$ alumnos) indicaron una mejora en la adquisición de conocimientos (media de 8,2) y en la satisfacción (media de 8,1)obtenida por parte del alumnado, que prefirió esta metodología a las exposiciones monográficas o el uso de simuladores por ordenador. En conclusión, la metodología aportada fue recibida con alto grado de satisfacción por su aplicación innovadora en los grupos pequeños.

Palabras clave: proyecto, innovación, docencia, trabajo colaborativo, coordinación, aprendizaje significativo

\section{Introducción}

La docencia universitaria se ha visto modificada para adaptarse a los nuevos criterios que marca el Espacio Europeo de Educación Superior (EEES) (European Association for Quality Assurance in Higher Education, 2005). De acuerdo a estas nuevas directrices es esencial que la enseñanza superior universitaria se convierta en un entorno dinámico y comunicativo que fomente el interés y la curiosidad, en este caso científica, para los alumnos que eligen unos estudios de grado. El proceso de aprendizaje permanente es un elemento clave para convertir Europa en la sociedad más competitiva y dinámica del mundo basada en el conocimiento. Es evidente que en la Universidad se debe enseñar, en primer lugar, un conjunto de saberes técnicos o conocimientos específicos para que los alumnos que salen de sus aulas sean capaces de ejercer con toda propiedad una profesión intelectual; pero, además, en los estudios correspondientes a las Ciencias de la Salud y en las de las Ciencias Básicas, es particularmente importante que la experiencia universitaria permita a los estudiantes desarrollar un pensamiento científico y que adquieran destreza en el razonamiento deductivo e inductivo. En este sentido, una vez establecidas las competencias que debe adquirir un alumno en relación con las materias o contenidos formativos específicos, se establecerán las actividades y experiencias que debe realizar para conseguir las mismas como resultado de su proceso de aprendizaje (Olmedo, 2013).

Unos de los aspectos más innovadores de los grados es la existencia de horas de seminarios en grupos reducidos que permiten trabajar en grupos pequeños aspectos específicos y más aplicados de las diferentes asignaturas (Blández, 2000). Así, los seminarios y / o talleres 
tienen como principal objetivo el construir conocimiento a través de la interacción y la actividad mediante sesiones monográficas supervisadas por el profesor. Este tipo de actividad formativa fomenta la participación, incita al debate entre compañeros y se permite el feedback o proceso de retroalimentación positiva en el aumento de conocimiento. Los seminarios son de gran importancia en la formación científica de los estudiantes. Pueden contribuir a promover un pensamiento más crítico y más coherente, para ayudar a resolver problemas y lleva a cabo aplicaciones prácticas de las teorías, para ampliar temas abordados en clase, fomentar la utilización de las fuentes bibliográficas, así como para estimular la motivación y el espíritu científico de los estudiantes (Cruz y Capa, 2005). Sin embargo, en muchas ocasiones no se obtiene todo el provecho que sería posible de estos seminarios al dedicar las sesiones a transmitir información de forma similar a las clases teóricas o corrigiendo actividades de forma bastante mecánica.

Otro aspecto a considerar dentro del proceso de aprendizaje son las actividades de aprendizaje fuera del aula. Dentro de este apartado de aprendizaje se engloban aquellas actividades que no requieren la presencia del profesor. Relacionado con los seminarios hay que destacar su preparación por medio de estudio y trabajo en grupo. El trabajo en grupo es una técnica educativa con un extraordinario potencial en el desarrollo del grupo y su rendimiento. Entre sus ventajas permite reducir el nivel de miedo a la clase y los alumnos se sienten libres para participar más activamente; se desarrollan actitudes de colaboración, sustituyendo a las de competitividad; aprenden más haciendo razonamientos en grupo que individualmente, ya que pueden llevar a un alumno a reducir la cadena de errores; el alumno es impulsado a expresarse y tomar iniciativas; y se practica la expresión oral, entre otros.

La Fisiología es una de las materias más integradoras de todas las que forman parte de las Ciencias de la Vida, dado que sus postulados recogen ideas y conceptos de otras disciplinas más instrumentales como la química, física, matemáticas, bioquímica, así como la biología molecular y celular. Así, la Fisiología Animal y Humana es una ciencia multidisciplinaria que se ocupa del estudio de cómo funcionan y cómo realizan las funciones los seres vivos. En este sentido, la físiología se centra en el carácter dinámico y funcional de lo que ocurre en los seres vivos. Las asignaturas de Fisiología se encuentran dentro diferentes grados como son el de Fisioterapia, Biología, Bioquímica y Medicina. El poder aplicar en esta materia la resolución de casos prácticos quizás suponga una estrategia para aumentar la motivación de los alumnos al ver una aplicación real de los conocimientos teóricos que van adquiriendo a lo largo del curso.

\section{Objetivos}

El objetivo principal del presente proyecto es introducir una nueva metodología activa de aprendizaje mediante la resolución de casos prácticos dentro de las horas de seminarios en grupo pequeño de las asignaturas de fisiología humana, y mejorar la coordinación entre el profesorado que imparte estas asignaturas en diferentes grados. Así, el objetivo engloba estimular la motivación y el pensamiento crítico de los alumnos mediante la resolución de casos reales, así como fomentar el trabajo en grupo y colaborativo y la adaptabilidad utilizando las tecnologías de la información y la comunicación (TIC) para la búsqueda de 
información y la presentación de los casos, consiguiendo además mejorar la capacidad de expresión oral.

\section{Desarrollo de la innovación}

La primera parte del proyecto supuso un proceso de coordinación entre los diferentes profesores que participaban en el proyecto y que imparten docencia de fisiología en todos los grados donde se imparte esta materia en la Universitat de les Illes Balears. A lo largo de los meses de junio y julio de 2018 se realizaron varias reuniones de trabajo entre los profesores implicados a fin de seleccionar casos prácticos que se adecuen al perfil de la asignatura y del alumnado en función del diferente grado donde se imparta la asignatura. Cada profesor en función del grado en el que imparte docencia hizo hincapié en los diferentes aspectos de su asignatura, indicando los aspectos básicos y diferenciales para poder establecer los objetivos prioritarios que el alumno debía entender y poner a punto de manera común y integrada.

Se prepararon diversos casos clínicos que consistían en un supuesto donde se aportaba a los alumnos unas variables médicas, bioquímicas o fisiológicas definidas y a partir de las cuales se podía "diagnosticar" la patología o alteración que el individuo presentaba. A partir de esta información, los estudiantes en grupos de 4-5 debían discutir el caso y elaborar una presentación para exponer, en primer lugar, la fisiología normal relacionada con la alteración presentada y luego, explicar la parte fisiológica que se veía afectada o alterada. De forma general, la forma de funcionar los seminarios consistió en exponer en una primera sesión el funcionamiento de los casos con un ejemplo preparado por el profesorado. Posteriormente, para la siguiente sesión, una semana antes de la msima se subió al campus digital (Aula Digital) una propuesta de caso práctico que los alumnos debían ir trabajando en grupo a lo largo de la semana. En la sesión de seminario se destinó la mitad de tiempo de la misma a resolver dudas relacionadas con el caso práctico propuesto con la ayuda del profesor y terminar de preparar una corta presentación en formato power point o similar. En la segunda parte de la sesión, algunos de los grupos realizaban la presentación del caso al resto de compañeros y se abría un tiempo de debate para dejar claro los diferentes aspectos que permitían la resolución del caso y la profundización del aspecto fisiológico. Las siguientes sesiones se realizaron siempre siguiendo el mismo procedimiento, la propuesta de caso era subida al Aula Digital con una semana de antelación y se realizaba en la sesión la resolución de dudas y la exposición por parte del alumnado.

Entre todos los profesores implicados se elaboró una rúbrica común para la evaluación del trabajo realizado por los alumnos, donde se valoraba punto a punto los aspectos relevantes que se querían alcanzar con la resolución de los casos.

Finalmente, se diseñó una encuesta evaluadora para que los alumnos la pudieran rellenar con el objetivo de conocer la aceptación de esta metodología para una mayor comprensión de los conceptos teóricos y actitudinales de los dos grados implicados y para poder detectar los aspectos más débiles para poder mejorar en un futuro. 


\section{Resultados}

Tras las reuniones iniciales con el profesorado implicado en las asignaturas de Fisiología Humana de los diferentes grados (Fisioterapia, Biología, Medicina, Bioquímica) se elaboró la rúbrica que incluía los diferentes ítems a ser calificados y que se presenta en la tabla 1. Cada uno de los ítems se valoró entre 0-3,9 puntos (novel), 4-6,9 (aprendiz), 7-8,9 y 9-10 (nivel experto). Se desarrolló una fórmula matemática para cubrir las competencias de la rúbrica, de tal manera que la calificación obtenida fue la de aplicar la siguiente ecuación:

$$
\text { Nota }=[(\mathrm{A} 1+\mathrm{A} 2+\mathrm{A} 3+\mathrm{A} 4)+2(\mathrm{~B} 1+\mathrm{B} 2+\mathrm{B} 3)] / 10 .
$$

Tabla 1. Rúbrica para la evaluación de los seminarios

\begin{tabular}{|c|c|c|c|c|}
\hline Ítems evaluables & Novel & Aprendiz & Avanzado & Experto \\
\hline $\begin{array}{l}\text { Organización y } \\
\text { estructura de la } \\
\text { exposición (A1) }\end{array}$ & $\begin{array}{l}\text { La calidad de los } \\
\text { materiales y } \\
\text { organización es } \\
\text { mala, sin orden } \\
\text { metodológico, } \\
\text { preámbulo o } \\
\text { introducción. } \\
\text { Falta de } \\
\text { optimización del } \\
\text { tiempo. }\end{array}$ & $\begin{array}{l}\text { La calidad de los } \\
\text { materiales y } \\
\text { organización es pobre, } \\
\text { sin un orden } \\
\text { metodológico claro. } \\
\text { Optimización del } \\
\text { tiempo regular. }\end{array}$ & $\begin{array}{l}\text { La calidad de los } \\
\text { materiales y } \\
\text { organización es buena, } \\
\text { siguiendo un orden } \\
\text { metodológico con } \\
\text { preámbulo e } \\
\text { introducción, y } \\
\text { conclusiones } \\
\text { argumentadas. Buena } \\
\text { optimización del } \\
\text { tiempo. }\end{array}$ & $\begin{array}{c}\text { La calidad de los materiales } \\
\text { y organización es excelente, } \\
\text { siguiendo un orden } \\
\text { metodológico que incluye } \\
\text { un preámbulo e } \\
\text { introducción, un buen } \\
\text { desarrollo y con } \\
\text { conclusiones bien } \\
\text { argumentadas. Perfecta } \\
\text { optimización del tiempo. }\end{array}$ \\
\hline $\begin{array}{l}\text { Apoyo gráfico } \\
\text { (medios y/o } \\
\text { materiales de } \\
\text { apoyo) (A2) }\end{array}$ & $\begin{array}{l}\text { El apoyo gráfico } \\
\text { está mal utilizado } \\
\text { y no interactúan } \\
\text { con el auditorio. }\end{array}$ & $\begin{array}{c}\text { El apoyo gráfico es } \\
\text { pobre. No interactúan } \\
\text { con el auditorio. }\end{array}$ & $\begin{array}{l}\text { Utilizan un medio } \\
\text { gráfico } \\
\text { adecuadamente pero } \\
\text { no interactúan con el } \\
\text { auditorio. }\end{array}$ & $\begin{array}{l}\text { Utilizan un medio gráfico } \\
\text { adecuadamente e } \\
\text { interactúan con el auditorio. }\end{array}$ \\
\hline $\begin{array}{c}\text { Uso apropiado } \\
\text { del lenguaje y de } \\
\text { la terminología } \\
\text { (A3) }\end{array}$ & $\begin{array}{l}\text { Uso inexistente o } \\
\text { inadecuado de la } \\
\text { terminología } \\
\text { científica. } \\
\text { Errores } \\
\text { gramaticales y } \\
\text { tipográficos } \\
\text { numerosos. }\end{array}$ & $\begin{array}{l}\text { Se usan términos de } \\
\text { manera incorrecta en } \\
\text { ocasiones. Algunos } \\
\text { errores gramaticales. }\end{array}$ & $\begin{array}{l}\text { Los conceptos y } \\
\text { términos se usan } \\
\text { correctamente. En } \\
\text { general sin errores } \\
\text { gramaticales. }\end{array}$ & $\begin{array}{l}\text { Los conceptos y términos } \\
\text { científicos se utilizan } \\
\text { correctamente y } \\
\text { constantemente. Sin errores } \\
\text { gramaticales o tipográficos. }\end{array}$ \\
\hline $\begin{array}{l}\text { Capacidad de } \\
\text { síntesis (A4) }\end{array}$ & No sintetizan. & Regular. & Buena. & Excelente. \\
\hline $\begin{array}{c}\text { Eficacia } \\
\text { comunicativa } \\
\text { (oratoria) }(\mathrm{B} 1)\end{array}$ & $\begin{array}{l}\text { El tono, claridad, } \\
\text { fluidez vocal y } \\
\text { manejo de } \\
\text { escenario es } \\
\text { malo. }\end{array}$ & $\begin{array}{l}\text { Solo algunos de los } \\
\text { aspectos siguientes son } \\
\text { buenos: tono, claridad, } \\
\text { fluidez vocal y manejo } \\
\text { de escenario. }\end{array}$ & $\begin{array}{l}\text { El tono, claridad, } \\
\text { fluidez vocal y manejo } \\
\text { de escenario es bueno. }\end{array}$ & $\begin{array}{l}\text { El tono, claridad, fluidez } \\
\text { vocal y manejo de escenario } \\
\text { es perfecto. }\end{array}$ \\
\hline Dominio del & Grado de & Grado de profundidad & Grado de profundidad & Grado de profundidad de la \\
\hline
\end{tabular}




\begin{tabular}{|c|c|c|c|c|}
\hline tema (B2) & $\begin{array}{l}\text { profundidad de la } \\
\text { investigación } \\
\text { bajo, presentando } \\
\text { nula seguridad y } \\
\text { dominio de los } \\
\text { conceptos clave. }\end{array}$ & $\begin{array}{l}\text { de la investigación } \\
\text { medio, presentando } \\
\text { poca seguridad y } \\
\text { dominio de los } \\
\text { conceptos clave. }\end{array}$ & $\begin{array}{l}\text { de la investigación } \\
\text { alto, presentando } \\
\text { seguridad y dominio } \\
\text { de los conceptos } \\
\text { clave. }\end{array}$ & $\begin{array}{l}\text { investigación muy alto, } \\
\text { presentando gran seguridad } \\
\text { y dominio de los conceptos } \\
\text { clave. }\end{array}$ \\
\hline $\begin{array}{l}\text { Calidad en la } \\
\text { defensa del } \\
\text { trabajo y a las } \\
\text { preguntas que se } \\
\text { planteen (B3) }\end{array}$ & $\begin{array}{c}\text { No responden a } \\
\text { los interrogantes } \\
\text { planteados tras la } \\
\text { presentación por } \\
\text { parte del profesor } \\
\text { y de los } \\
\text { compañeros }\end{array}$ & $\begin{array}{c}\text { Responden } \\
\text { insuficientemente a los } \\
\text { interrogantes } \\
\text { planteados tras la } \\
\text { presentación por parte } \\
\text { del profesor y de los } \\
\text { compañeros }\end{array}$ & $\begin{array}{c}\text { Responden a los } \\
\text { interrogantes } \\
\text { planteados tras la } \\
\text { presentación por parte } \\
\text { del profesor y de los } \\
\text { compañeros }\end{array}$ & $\begin{array}{l}\text { Responden perfectamente a } \\
\text { los interrogantes planteados } \\
\text { tras la presentación por } \\
\text { parte del profesor y de los } \\
\text { compañeros. }\end{array}$ \\
\hline
\end{tabular}

En las mismas reuniones entre el profesorado implicado se desarrolló la encuesta para evaluar el grado de satisfacción del alumnado en relación a la metodología que se implantó, y que incluían las siguientes cuestiones que debían ser puntuadas del 0 al 10 , siendo el 0 estar en total desacuerdo y el 10 estar totalmente a favor:

1. ¿Cómo evalúas el procedimiento seguido a lo largo de los seminarios?

2. ¿La realización de los seminarios te ha permitido adaptarte a la realización de exposiciones orales de manera gradual mejorando a lo largo de los mismos?

3. ¿La realización de un mismo caso clínico por algunos de tus compañeros te ha permitido evaluar las diferencias entre los grupos?

4. ¿Cómo valoras la participación de tus compañeros en la realización de preguntas?

5. ¿Te parece adecuada la realización de estos seminarios para reforzar y/o profundizar algunos conceptos de las clases magistrales?

6. ¿Preferirías la realización de un seminario monográfico centrándose en la explicación de un tema concreto como se ha realizado clásicamente? / ¿Preferirías el uso de simuladores de fisiología por ordenador?

7. A nivel global, ¿la forma de evaluar los seminarios te parece correcta?

8. Valora de 1 a 10 los siguientes aspectos en relación a los seminarios:

a) Claridad en la organización

b) Satisfacción de expectativas

c) Utilidad para la comprensión y asimilación de conceptos

d) Utilidad para incentivar el trabajo autónomo

e) Utilidad para promover el autoaprendizaje

f) Utilidad para la adquisición de destrezas para la resolución de problemas 
9. Finalmente, se dejó una novena pregunta para respuesta libre en la cual el alumnado podía incluir los comentarios que creyese convenientes en relación a una mejora de la estructura de la metodología seguida en los grupos pequeños o seminarios.

Los resultados obtenidos en relación a las cuestiones se representan en la figura 1. Los alumnos que participaron en la experiencia fueron la totalidad del grupo en todos los grados, ya que se tratan de actividades de asistencia obligatoria, ascendiendo a un total de 174 alumnos ( $\mathrm{n}=46$ en Fisioterapia, $\mathrm{n}=45$ en Biología, $\mathrm{n}=25$ en Bioquímica y $\mathrm{n}=58$ en Medicina). Se puede observar que las puntuaciones promedio a cada una de las preguntas del cuestionario se encuentran por encima de valores de 7,5, con excepción de la pregunta en relación a la valoración de los compañeros a la hora de realizar preguntas, en la que el valor obtenido es de 5,7. Adicionalmente, la cuestión en relación a si prefieren un cambio de metodología hacia un tipo de presentación monográfica en lugar de la metodología de casos prácticos recibió una puntuación de 3,3. Entre los comentarios de respuesta libre en el último apartado del cuestionario, se indica que los seminarios son útiles (más que prácticas de laboratorio o la realización de simulaciones con ordenador) y dinámicos con casos clínicos interesantes en los que se aprende siempre algo y que fomentan la participación en clase, siendo un modelo que debería ser incluido en otras asignaturas ya que incentiva el aprendizaje, fomenta el trabajo autónomo y la adquisición de conocimientos; adicionalmente, ayudan a coger confianza y que no cambiarían la metodología. También valoran positivamente la primera sesión a modo ejemplo realizada por el profesor. Por otro lado, indican que se podría incentivar más la interacción oyente-exposición (no por parte del profesor, sino con el resto de compañeros, aunque el mismo alumno/a indica que es difícil obligar a los alumnos a dicha interacción), que podrían conocer los temas de los otros grupos para preparar preguntas interesantes, alargar el tiempo de exposición y discusión de cada uno de los casos para profundizar más aún en la fisiología e incluir más casos prácticos a resolver para no repetir alguno de ellos.

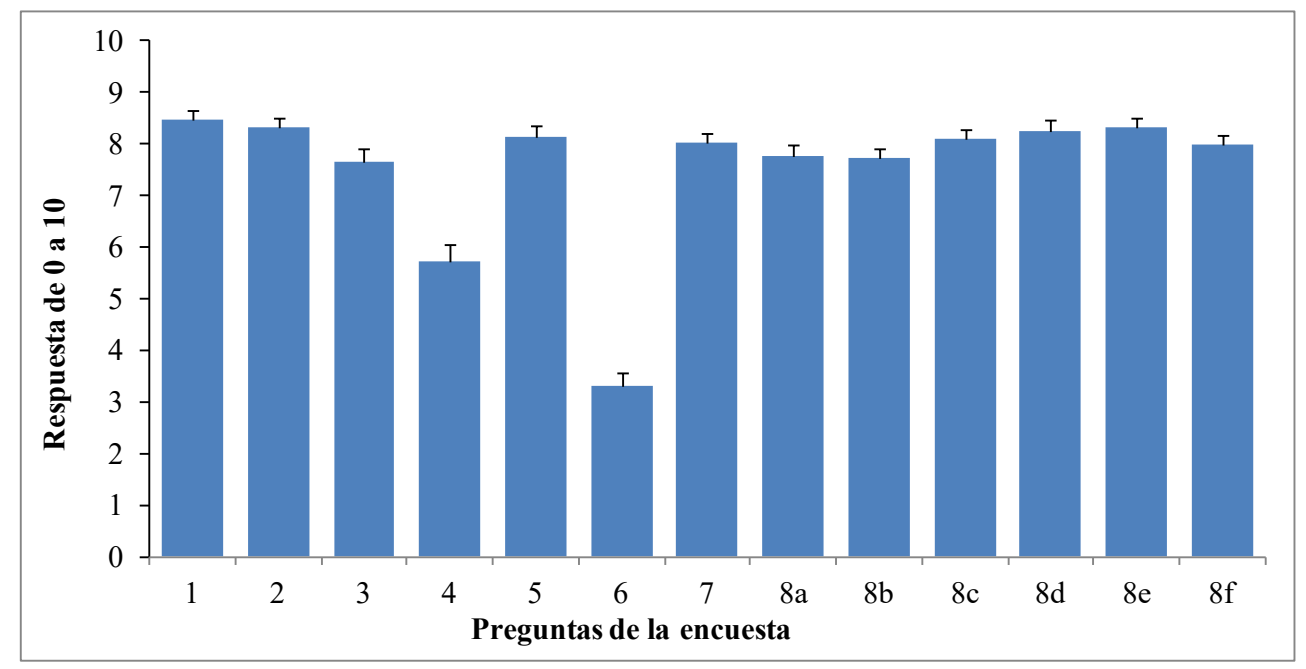

Figura 1. Resultados de los alumnos que han cursado Fisiología Humana según la encuesta realizada sobre la metodología utilizada en los seminarios en grupos pequeños sobre la resolución de casos prácticos. Los números indican cada una de las preguntas del cuestionariocontestado por los alumnos de los diferentes grados. 
Finalmente indicar que las valoraciones fueron ligeramente diferentes atendiendo al grado, así, la valoración positiva de la metodología implantada en los grupos pequeños fuer mayor en el grado de Fisioterapia en comparación con el resto, siendo el grado de Medicina el que presentó valores menores en las encuestas.

\section{Conclusiones}

La introducción de la resolución de casos prácticos como nueva metodología activa de aprendizaje durante las horas en grupo mediano de las asignaturas de fisiología humana fue valorada muy positivamente por el alumnado, y permitió una mejora en relación a la coordinación y, por tanto en la calidad docente, entre el profesorado que imparte estas asignaturas en diferentes grados (Fisioterapia, Biología, Bioquímica y Medicina). La resolución de casos clínicos reales mediante la metodología descrita permitió motivar a los alumnos, los cuales profundizaban en el caso práctico y la fisiología relacionada, realizando un trabajo en grupo colaborativo de una manera crítica que ayudó a una mayor confianza del alumno ante el hecho de presentarse ante un auditorio y una mejora notable en la capacidad de expresión oral y el uso de terminología adecuada. Por otro lado, un grupo minoritario de alumnos indicó que les gustaría que en un mismo grupo no se repitiera el mismo caso clínico y conocer el caso con anterioridad para preparar cuestiones a realizar; sin embargo, la duplicación de los mismos tenía como objetivo la autovaloración y crítica constructiva por parte del mismo alumnado del trabajo realizado con respecto a un caso práctico; además, la resolución con anterioridad a su presentación no permitiría seguir la metodología planteada incialmente. La aplicación de esta metodología en otras asignaturas y grados universitarios podría ser posible mediante el establecimiento de problemas iniciales (en similitud con un caso clínico práctico) y el desarrollo de una propuesta de solución/explicación del problema inicial planteado por parte del alumnado en la que tenga que investigar y relacionar conceptos incrementando la autonomía y crítica en la búsqueda de respuestas, además de conseguir un aprendizaje significativo. Hay que tener en cuenta que los resultados arrojan una aceptación elevada por parte de alumnado en la aplicación de esta metodología versus a otras metodologías más clásicas como son la realización de exposición sobre un tema monográfico.

En conclusión, la resolución de casos prácticos como metodología activa de aprendizaje en las asignaturas de Fisiología Humana en diferentes grados ha sido valorada muy positivamente prefiriéndola a las clásicas exposiciones monográficas.

\section{Referencias}

BLANDEZ ÁNGEL, J. (2000). La investigación acción: un reto para el profesorado : guía práctica para grupos de trabajo, seminarios y equipos de investigación. Barcelona : INDE, $2^{\mathrm{a}}$ ed.

CRUZ, A., CAPA, A. B. (2005). Nuevas claves para la docencia universitaria en el Espacio Europeo de Educación Superior. Ed. Narcea.

EUROPEAN ASSOCIATION FOR QUALITY ASSURANCE IN HIGHER EDUCATION (2005). Criterios y Directrices para la Garantía de Calidad en el Espacio Europeo de Educación

Superior. 
$<$ https://enqa.eu/indirme/esg/ESG\%20in\%20Spanish_by\%20ACPUA.pdf $>$ [Consulta: 8 de marzo de 2019]

OLMEDO MORENO, E. M. (2013). "Enfoques de aprendizaje de los estudiantes y metodología docente: Evolución hacia el nuevo sistema de formación e interacción propuesta en el EEES" en Revista de Investigación Educativa, vo. 31, p. 411-429. 